\title{
Advances in STM Design and Instrumentation
}

\author{
R. Wiesendanger, D. Anselmetti \\ and H.-J. Güntherodt
}

\author{
Institute for Physics, University of Basel, Switzerland
}

Scanning tunneling microscopy and related scanning techniques have led to remarkable progress in understanding the structures of surfaces. This progress has mainly resulted from improvements in instrument design and operational reliability.

A few years ago, experimentalists were proud to show the atomic resolution capability of their instruments in all kinds of environments such as air, inert gases, liquids, ultrahigh vacuum (UHV), and low temperatures. Today, scanning tunneling microscopes are being constructed to work under extreme conditions, e.g. at the lowest possible background pressure, at the lowest possible temperatures and at the highest possible magnetic fields. For instance, Fein et al. [1] have designed a STM that operates at temperatures down to $400 \mathrm{mK}$ in magnetic fields up to 8 tesla. In the authors' laboratory, a UHV compatible STM has been built that works in the in the $10^{-12}$ mbar range (Fig. 1).

Combinations of STM with other microscopic or analytical techniques have also been realized including STM with optical, electron and field ion microscopes as well as with almost all known conventional surface analytical techniques. Finally, by changing the probing mechanism between the tip and the

Dr. Roland Wiesendanger, on the research staff at the Institute for Physics, University of Basel, Klingelbergstrasse 82, CH-4056 Basel, Switzerland, received his Ph.D. in 1987 from the University. Working with STM since 1984 , his research activities include the application of STM combined with other analytical tools to surface science and nanotechnology.

Mr. Dario Anselmetti received his diploma in experimental physics in 1987 from the University of Basel and is currently working towards a Ph.D. His research focuses on STM studies of various metals and on the development of a low temperature STM device.

Dr. Hans-J. Güntherodt, professor of physics since 1974 in the Institute for Physics, received his Ph.D. degree in 1967 from the ETH Zürich where he remained to continue his research into liquid metals until he moved to Basel. His principal research interests lie in condensed matter physics: metallic glasses, graphite intercalation compounds, and more recently tunneling microscopy and nanometre scale science. sample surface, several STM-related scanning probe microscopes have been invented, offering the chance to measure various kinds of surface properties with a spatial resolution in the nanometer range.

Fig. 1 - (a) Schematic drawing of the STM unit used in the authors' laboratory for surface studies of metals and semiconductors in UHV. The horizontal movement of an electromagnetically driven wedge is transformed into a vertical movement of the sam. ple stage which carries the sample holder and the horizontally mounted sample. The scanning unit or head rests on three ball bearings and can be flipped backwards, giving easy access to the sarnple and tip.

(b) The STM unit described in (a) is mounted on top of a stack of five stainless steel plates separated by Viton rubber elements. The stack is supported by the inner platform of a two-stage spring system combined with eddy current damping. The whole unit (STM plus vibration isolation system) is inserted into the UHV chamber through its bottom flange using an elevator.
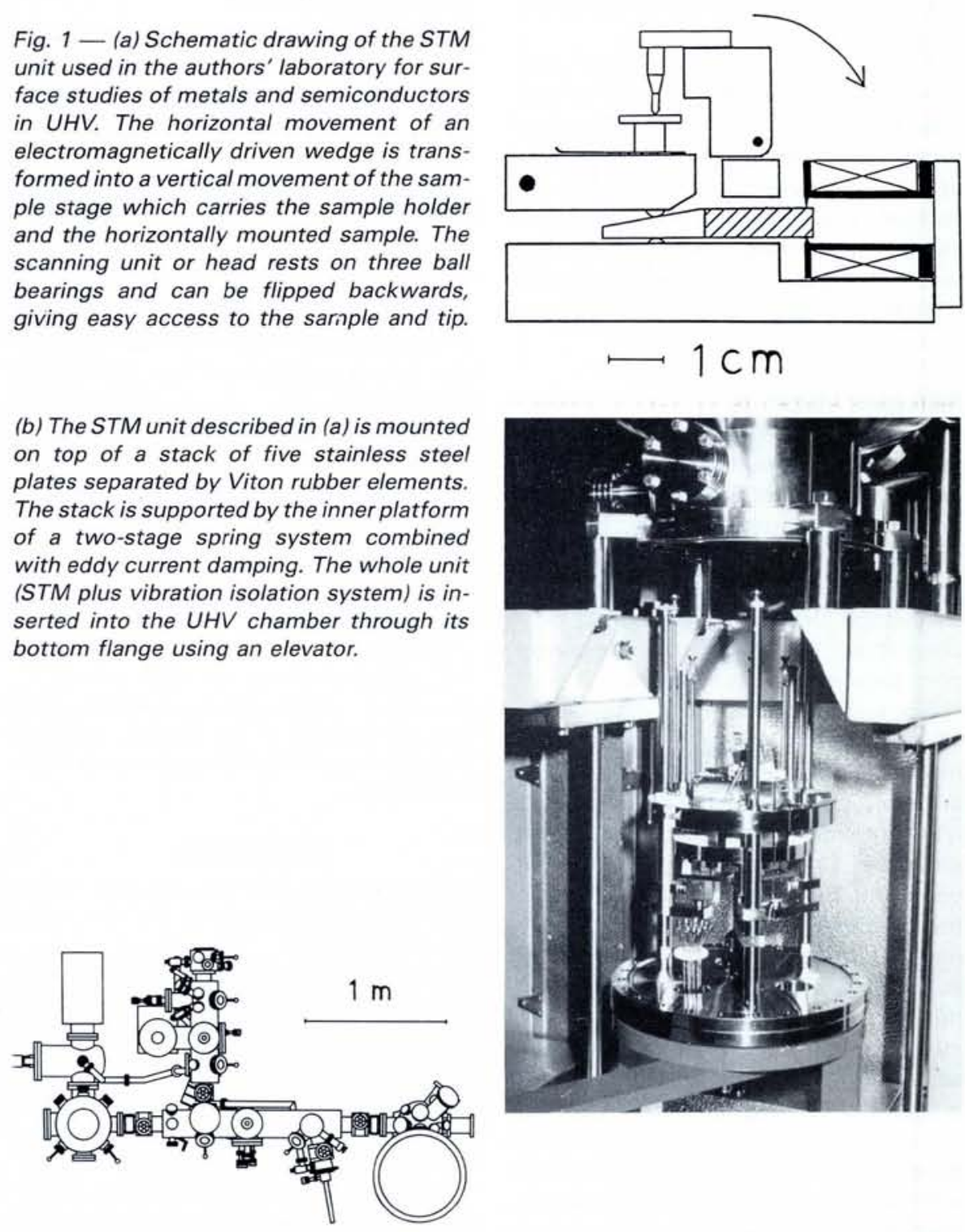

(c) Schematic drawing (top view) of the entire UHV surface analysis system consisting of separate chambers (from right to left) for surface analysis, sample transfer, sample preparation and STM imaging. Preparation facilities include resistive and electron beam heating, ion etching, evaporation, gas inlet and high pressure gas reaction. Conventional surface analysis techniques such as LEED, SEM/SAM, XPS and UPS are used for additional characterization of the surfaces and for obtaining complementary information. The whole UHV system is mounted on sixteen air pads for vibration isolation. 
These demands automatically lead to an increase in the size and to a more open construction, thereby decreasing the rigidity. An efficient vibration isolation system has therefore to be added. In UHV, the best choice may still be a two stage spring system combined with eddy current damping (Fig. 1), as originally used by $\mathrm{G}$. Binnig, $\mathrm{H}$. Rohrer and coworkers for their second generation STM instruments. Additionally, or as an alternative, a stack of metal plates separated by Viton damping elements may be used, as was first successfully demonstrated for the "pocket-size" STM [4]. Commercially available vibration isolation systems consisting of pneumatic damping elements are used in conjunction with STM's working in al kinds of environments.

\section{Probe Positioning}

A piezoelectric walker (the "louse") was used in the early days of STM for the coarse approach of the sample towards the probe tip. Today, piezoelectric driving elements such as "inchworms" or electromagnetic walkers (Fig. 1) are implemented. To further increase the rigidity of the STM unit, differential screws driving either a reduction lever or a differential spring system have proven to be very successful in many STM designs (Fig. 2). Finally, replacing the piezoelectric tripod scanning unit by a tube scanner [5] affords the opportunity to construct even smaller and more compact STM's with higher resonance frequencies. However, piezoelctric hysteresis, creep and crosstalk between the three possible directions of movement still remain a problem, particularly for large area scans (several square micrometers).

\section{Electronics}

Advances in the mechanical aspects of STM design have been accompanied by advances in electronics and computerised automation. A standard STM electronics and data acquisition system nowadays consist of a computer controlled approach circuit, a bias voltage supply for the sample, using either a battery or a computer controlled digitalto-analogue converter (DAC), a preamplifier with a gain of $10^{6}-10^{9}$ V/A, a logarithmic amplifier, a feedback loop realized either with an analog integrator and a proportional amplifier or by digital feedback, a scan generator provided by synchronized ramp generators or computer controlled DACs, high voltage amplifiers for the amplification of the scan and feedback signals applied to the $x, y$ and $z$-axis piezoelectric drivers,

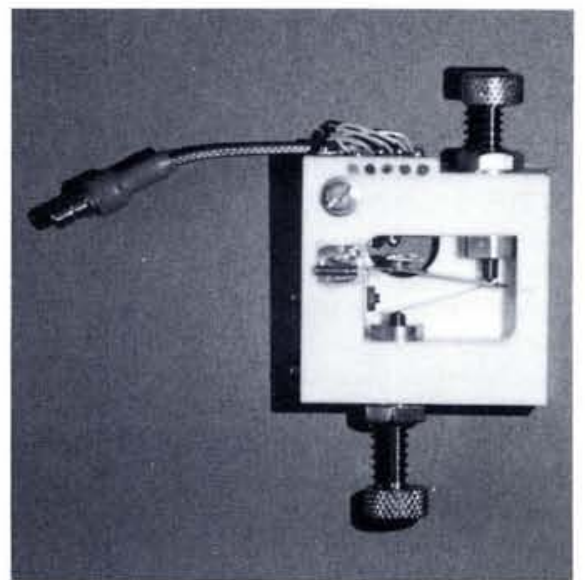

COARSE APPROACH
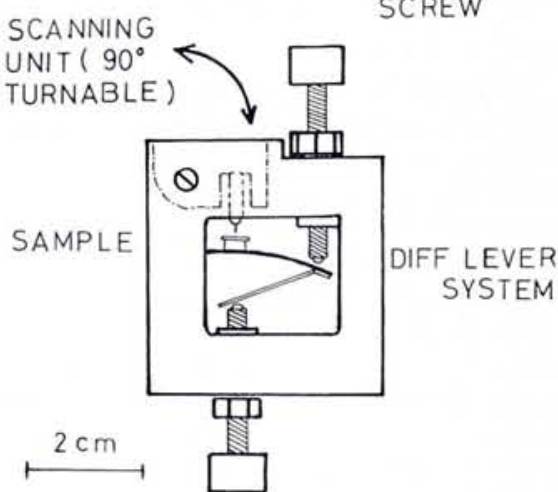

FINE APPROACH

SCREW

Fig. 2 - (a) Photograph and (b) schematic drawing of a rigid STM unit used in the authors' laboratory for surface studies in air, in inert gas atmospheres and at low temperatures. The approach mechanism is based on a differential lever system consisting of two levers with different spring constants. The sample, mounted on the lever with the higher spring constant, is moved towards the tip by reducing the tension of this lever with the coarse approach screw. Fine adjustment is achieved by bending the lever with the lower spring constant with the fine approach screw.

A single piezoelectric tube scanner with the tip holder is fixed to a scanning head that can be rotated through $90^{\circ}$ to allow the tip and sample to be changed. The microscope, although rigid enough to be operated at low temperatures without an additional damping system, can also be mounted on top of a stack of five stainless steel plates separated by Viton rubber elements, or supported by a commercially available antivibration table.

and several analogue-to-digital converters digitizing the desired signals (feedback signal, current, etc.). A "sample and hold" circuit is desirable for the implementation of all kinds of spectroscopic capabilities. A very low level of electronic noise is important, including a minimum amount of pickup of electrical disturbances from external sources such as switches.

\section{Probes}

The most important requirement for future progress in STM technology may well be improved control of the atomic scale structure of the probe tip. Producing STM tips by simply cutting a Pt-Ir wire or by electrochemically etching $\mathrm{W}$ wire, sometimes combined with an additional in-situ treatment such as electron bombardment, field emission or the application of voltage pulses, undoubtedly yields satisfactory images of atomic surface structures. However, particularly for the interpretation of spectroscopic information, better knowledge of the atomic cluster at the front end of the tip and its electronic states, which determine the tunneling current, is desired. Well defined single atom tips [6] used for STM investigations might provide one solution.

\section{Concluding Remarks}

Besides the progress that has been made in STM instrumentation, many novel but related scanning probe micro- scopies have been invented during the last few years. Their development has profited greatly from the experience gained by working with STM. These novel microscopies offer the possibility of studying the surfaces of materials which cannot be imaged using STM, e.g. bulk insulators. They also provide additional information such as stray magnetic field distributions, charge distributions, frictional properties and thermal profiles to mention only a few possibilities.

The family of scanning probe microscopies has already led to a new understanding of the properties of matter on the nanometre scale. Future progress in the design and instrumentation of scanning probe microscopes will at least partly arise from miniaturization, e.g. "STM's on a chip" [7], leading to micrometre and even nanometre-sized probes for atomic scale properties.

\section{REFERENCES}

[1] Fein A.P., Kirtley J.R. and Feenstra R.M., Rev. Sci. Instrum. 58 (1987) 1806.

[2] Pohl D.W., IBM J. Res. Rev. 30 (1986) 353.

[3] Yulk Y. and Silverman P.J., Rev. Sci. Instrum. 60 (1989) 165.

[4] Gerber Ch., Binnig G., Fuchs H., Marti O. and Rohrer H., Rev. Sci. Instrum. 57 (1986) 221.

[5] Binnig G. and Smith D.P.E., Rev. Sci. Instrum. 57 (1986) 1688.

[6] Fink H.W., Phys. Scripta 38 (1988) 260. [7] Albrecht T.R., Ph.D. Thesis (Stanford University) 1989. 